\title{
L'interpretation sociale, une interdiscipline face à ses théories
}

\author{
Hildegard Vermeiren \\ Hogeschool Gent
}

This article intends to contribute to the theoretical status questionis concerning social interpreting. Starting point is the very name of the discipline, which refers to sociology as well to communication and linguistics. The purpose is to examine how each of these research areas offers a number of concepts relevant to social interpreting, in particular those concepts referring to the category "subject". The path that we have chosen is twofold. First we explore the development of each of these disciplines and the ensuing creation of relevant concepts for our topic of investigation. Next, we analyse how these three sciences have learned and borrowed from each other and what the relevance of this can be for research into social interpreting. We conclude the article with a schematic overview showing the corresponding relevant concepts placed next to each other.

\section{Un état de la question interdisciplinaire}

Notre point de départ est le nom même de la discipline appelée interprétation sociale. Ce nom composé nous révèle sa nature interdisciplinaire: la sociologie s'y mélange avec la traductologie et la linguistique. Comme l'espace limité d'un article ne permet pas d'approfondir toutes les connexions entre les théories de ces trois disciplines, nous nous limitons à un bref panorama de quelques-uns de leurs concepts-clés, notamment ceux qui concernent le sujet. Un tel inventaire non seulement permet de mieux saisir et de clarifier ces concepts, mais aussi de mieux fixer des points d'ancrage pour des réflexions ultérieures.

En guise de test nous avons relevé les concepts sociologiques, traductologiques et linguistiques dans les pages théoriques de deux ouvrages classiques de l'interprétation sociale, notamment Liaison Interpreting. A Handbook de Gentile, Ozolins et Vasilikakos (1996) et Interpreting as Interaction de Wadensjö (1998). Dans la partie théorique du premier apparaît une grande majorité de termes et concepts appartenant à la sociologie, face à une stricte minorité de termes et concepts traductologiques ou linguistiques. Le caractère interdisciplinaire de l' interprétation sociale ne ressort donc pas clairement de cet ouvrage. Dans l'ouvrage de Wadensjö il y a un meilleur équilibre entre les concepts sociologiques et traductologiques, mais la théorie linguistique y est également peu visible. Ce deuxième ou- 
vrage représente cependant nettement mieux le caractère multidisciplinaire de l'interprétation sociale.

Dans le cadre d'un état de la question, il est sans doute intéressant de retourner aux origines même des concepts. La première voie à parcourir sera celle empruntée par la sociologie afin de se rapprocher de l'étude du langage. Ce départ nettement sociologique sera utilisé comme une sorte de paradigme auquel nous confronterons ensuite les deux autres disciplines. La deuxième voie sera celle de la communication dans son rapprochement de l'interprétation d'abord et de la sociologie ensuite; la troisième voie sera celle qu' a empruntée la linguistique pour se rapprocher des phénomènes langagiers du type parole. Notre but est d'élaborer un tableau synoptique résumant les concepts correspondants des trois disciplines.

\section{Evolution de la sociologie vers la communication et l'étude de l'interprétation: concepts de base}

La voie parcourue par la sociologie peut être résumée en six étapes, qui nous permettent de cerner l'apparition d'une série de concepts fondamentaux.

Le point de départ est le sociologue allemand Weber, qui lance une psychosociologie centrée sur l'individu et sa rationalité. Sa théorie prône la liberté, la créativité de l'individu face à la société. Un concept central y est l'action. Les actions humaines ont une signification, elles s'expliquent par les raisons et les intentions de leurs agents. Le but de la sociologie est donc d'étudier la signification des actions humaines. Weber distingue deux perspectives possibles dans le sens de l'action: le sens donné par l'agent de l'action à sa propre action, et d'autre part le sens donné à cette même action par celui qui l'observe de l'extérieur (Waters 1994: 15-20).

Un contemporain de Weber, Simmel, y ajoute à son tour quelques concepts importants. Il s'intéresse particulièrement aux côtés formels de l'action humaine, plus particulièrement aux différents cadres issus des différences quantitatives dans l'interaction entre les membres d'une société. L'apprentissage de ces cadres s'appelle la sociation (à ne pas confondre avec la socialisation). Sur le plan quantitatif, il distingue trois niveaux: l'individu, le petit groupe et la masse. Ce qui l'intéresse particulièrement est le fonctionnement des dyades (deux individus en interaction) et des triades (trois individus en interaction). Dans ce dernier cas, il y a trois formations possibles, selon la nature du troisième élément. Le rôle de médiateur entraîne la neutralité et exempte de la responsabilité de résoudre les conflits. Le rôle d'arbitre entraîne la responsabilité de trancher les conflits. Finalement le tertium gaudens est une tierce personne tirant profit d'un conflit entre les deux premières (Wolff 1964: 105-177) (Waters 1994: 2022).

Un psychosociologue américain, Mead, reprend les concepts d'action et interaction pour spécifier que la faculté du langage est ce qui différencie l'interaction humaine de l'interaction animale. La psychosociologie aboutit 
ainsi à un courant de recherche appelé interaction symbolique. C'est justement la faculté du langage qui permet à l'homme de structurer son soi (en anglais 'self'); en d'autres mots, c'est par l'usage du je et du moi dans son interaction verbale avec d'autres personnes, qu'il devient un agent. Mead souligne que ce processus de structuration du soi se réalise à travers l'adoption de rôles, ce que l'être humain apprend à faire dès son enfance. De cette façon, Mead (1970: 135-222) reprend l'idée de la sociation prônée par Simmel (Waters 1994: 22-26).

L'un des grands représentants de ce même courant est le psychosociologue canadien Goffman. Ses idées sont devenues une espèce de modèle généralement accepté par l'interprétation sociale. Le centre d'attention de ses recherches est également le soi, particulièrement dans sa relation avec les autres, qui constituent son audience. Pour mieux cerner ce domaine, Goffman fait usage d'une métaphore dramaturgique, qui prévoit pour tout cadre (en anglais 'frame') un ensemble de rôles, qui peuvent être soit des rôles standard, soit des rôles déviants. Il y a aussi des rôles appuyant la pièce proprement dite, mais se situant en fait dans la périphérie de l'action théatrale même. Ce sont des rôles de non-personne: par exemple les rôles joués par le personnel technique (gardiens de sécurité, coiffeurs, grimeurs, etc.), par des informants ou des intermédiaires (en anglais 'gobetween') (1959: 150-153). Un interprète serait donc une sorte d'intermédiaire. Ces non-personnes sont soit présentes à un vraie performance d'agents en interaction, soit elles opèrent depuis les coulisses (1959: 152-153). Goffman s'intéresse aussi au contrôle exercé par le soi de l'image qu'il donne de lui-même à son audience (1959: 208-237). Il est évident qu'un intermédiaire doit cultiver son image, sa face. Un autre concept est l'alignement (en anglais 'footing') des différents participants dans une interaction face à face (1981: 128) Cette capacité d'alignement concerne aussi bien l'alignement du soi sur les autres que celui des autres sur le soi, en d'autres mots, il entraîne la capacité du soi d'assigner des positions et statuts différents aux agents et l'audience d'une interaction. Goffman distingue le locuteur, le destinataire ratifié adressé ou le destinataire ratifié non-adressé, ainsi que les récipiendaires non-adressés (1981: 131-137). Il relève également la capacité humaine de faire comme si certaines personnes n'étaient pas présentes, en leur donnant (unilatéralement ou non) un statut de nonpersonne (1959: 152-153). Pour raffiner davantage l' alignement, il continue son analyse du locuteur, qu'il subdivise du point de vue social en auteur (qui choisit le contenu du message ainsi que les émotions à transmettre) et principal (qui définit sa position par ce qu'il dit). Il y ajoute un rôle analytique (non social) qu'il appelle l'animateur (qui transmet une énonciation sans transmettre un message propre), qu'il illustre d'ailleurs par l'exemple d'un traducteur simultané (1981: 144-147). Il n'approfondit cependant pas cette dernière question: est-ce que le traducteur simultané est une sorte d'intermédiaire, d'animateur, donc, par définition, une non-personne?

Gumperz, un anthropologue linguistique américain, oriente ces recherches vers l'interaction spécifiquement verbale et se rapproche de la communication. Il souligne l'importance d'avoir des compétences com- 
municatives très développées: elles constituent un capital à la fois symbolique et social (1982: 5). De plus, notre compétence langagière contribue à la construction et au maintien de notre identité (1982: 7). Dans ses essais il prône le relativisme, avançant que notre culture détermine notre façon de percevoir les choses. Le contexte culturel est effectivement un facteur très important dont tout interprète doit tenir compte, surtout en travaillant pour des personnes ethniquement différentes. La flexibilité communicative est une compétence qui doit permettre de vaincre les obstacles à la communication. Tout ce qu'il avance sur la perception et la flexibilité est particulièrement fertile pour les recherches en interprétation en général, et plus spécifiquement, pour l'interprétation sociale.

Finalement, un autre sociologue américain, Anderson (2002: 206217) mélange la sociologie et l'étude de l'interprétation dans une réflexion sur le rôle spécifique de l'interprète. Un traducteur est pour lui un bilingue qui travaille par écrit, dans l'isolement social. Un interprète, en revanche, est un bilingue qui intervient oralement dans des situations sociales sur lesquelles il a un impact certain. Le modèle qui inspire Anderson est clairement celui de l'interprète de liaison faisant un travail bidirectionnel face à face avec des partenaires appartenant à des groupes ethniques ou sociaux différents. Dans son modèle de la communication, le producteur et le consommateur monolingues sont assistés par un interprète, qui fait fonction de pivot en assurant une communication bilingue bidirectionnelle.

Anderson réfléchit d'abord sur les aspects linguistiques de la communication et constate un clivage quant aux compétences des interprètes. Comme toutes les personnes bilingues, les interprètes ont une langue dominante et une autre langue qu'ils connaissent moins bien. La traduction vers la langue dominante sera en principe de meilleure qualité. Il signale aussi qu' un interprète a tendance à s'identifier davantage avec les personnes qui ont la même langue dominante que lui.

Ensuite il analyse le conflit de rôle vécu par l'interprète durant le face à face avec ses clients. En premier lieu, il doit servir deux clients en même temps, ce qui peut causer un stress non négligeable. En deuxième lieu, il se voit confronté à des ambiguïtés. Il y a en effet un manque de clarté sur son rôle exact (simple écho, médiateur, conseiller, intermédiaire, allié ?). Il sait que s'il fait un travail bidirectionnel il est tenu à être fidèle aussi bien à sa source qu'à sa cible. Pour éviter ce conflit de rôle, justement, on préfère dans les conférences qu'il y ait un interprète différent (natif si possible) pour chaque langue cible. L'interprète de liaison, en revanche, ne peut pas travailler pour une seule cible et limiter sa fidélité rien qu'à celleci. Il doit chercher un équilibre entre ses deux clients, leurs langues et cultures, assumant ainsi un rôle de non-partisan (2002: 213). Pour expliquer ce rôle, Anderson reprend le rôle de médiateur défini par Simmel, soulignant que malgré sa neutralité, l'interprète peut manipuler considérablement le message et donc l'audience. Finalement il parle du pouvoir de l'interprète, qui lui est donné par le fait d'être bilingue, et de diriger l'interaction. Lorsque l'interprète filtre la communication, il peut en même temps contrôler l'interaction au sein de la triade. 
Ce parcours conceptuel à travers la sociologie, vers la science de la communication et l'interprétation, nous a fourni une série de concepts. Le modèle conceptuel généralement accepté est celui de Goffman, enrichi avec d'autres apports. Il permet de distinguer de façon nette l'interprétation sociale et l'interprétation de conférence.

L'interprète social travaille en petit groupe, concrètement, dans une dyade de clients qui, grâce à sa présence autour de la table, se transforme en triade. L'interprète expose son identité, son soi, en faisant un travail face à face, bidirectionnel, devant une audience (généralement hétérogène) constituée par deux personnes (à statuts différents), dont il contrôle l'interaction. Cette situation l'oblige à faire usage de différentes stratégies pour maintenir sa neutralité.

L'interprète de conférence, en revanche, travaille pour un grand groupe de personnes (généralement homogène), auquel il ne s'expose pas en tant que soi, puisque le face à face et le travail à proximité physique sont remplacés par l'isolement et la sécurité d'une cabine d'où il produit une traduction unidirectionnelle vers sa langue native. Il a moins besoin de stratégies pour maintenir sa neutralité, mais il contrôle moins l'interaction des participants.

\section{Evolution de la communication et l'interprétation vers la sociologie: concepts de base}

La voie parcourue par les études de l'interprétation diffère de celle de la sociologie. Il s'agit d'un sous-domaine de la traductologie, qui est, à son tour, une sous-discipline de la science de la communication. Néanmoins nous avons remarqué que dans les ouvrages théoriques sur l'interprétation le lien avec la théorie de la communication reste peu explicite. Comme le concept de communication n'apparaît même pas dans la carte des memes de l'interprétation (terme lancé par Chesterman en 1997 pour parler des idées ou pratiques qui circulent à l'intérieur d'une discipline) élaborée par Pöchhacker (2002: 60), il nous semble utile de l'approfondir. Kondo (2003: 77), d'autre part, signale que le schéma de la communication interlinguale constitue l'essence de l'interprétation.

Il s'agit de disciplines plus jeunes que la sociologie. Leur origine se situe dans les textes fondationnels écrits sur la communication au début du vingtième siècle, entre autres, par Shannon et Weaver (1947). Le modèle général de la communication dont nous parlent ces auteurs est du type linéaire: il permet qu'un émetteur, grâce à l'usage d'un code (verbal ou autre) envoie à travers un canal un message à un récepteur, qui le décode. C'est un modèle simpliste, qui ne tient pas compte du contexte, qui n'a pas de dimensions psychologique ou sociologique, qui n' attribue qu'un rôle passif au récepteur, qui ne prévoit aucune boucle de rétroaction, ni, bien sûr, d'interprète (1971: 260-266).

Ce modèle est adapté à la communication verbale par Jakobson (1963: 206-220), qui prévoit outre le destinataire, le destinateur, le message 
et le code d'autres dimensions telles que le contact et le contexte. Jakobson ne prévoit pas de place pour la traduction ou l'interprétation, même s'il s'intéresse également à ces deux disciplines (1963: 78-86).

Ingram (1985: 97-102) développe un modèle sémiotique pour l'interprétation simultanée en langage gestuel. Il s'agit d'un modèle à sept facteurs: la source, l'encodage, l'interprète, le décodage, le récepteur, le contexte et le bruit, situés sur ou autour du canal de transmission. C'est le premier modèle qui contemple la figure de l'interprète (1985: 98), qui est une sorte de canal de communication entre la source et le récepteur. Ingram relève le fait que pour comprendre le message d'une source, l'interprète doit maîtriser de nombreux codes, non seulement les codes verbal et gestuel.

Kirchoff (1976: 21-27) développe un modèle nettement plus avancé, qui représente l'interprétation entre trois personnes et deux langues. Ce modèle a été généralement accepté par la communauté internationale des interprètes (Kondo 2003: 78). Kirchoff distingue les partenaires primaires - l'émetteur et le récepteur- qui sont monolingues, et l'interprète, qui est bilingue et biculturel, et qui agit en tant que médiateur. L'émetteur n'a aucun contrôle sur le message tel qu'il est transmis par l'interprète vers le récepteur; il doit avoir confiance dans sa compétence et sa volonté de coopération. La même chose vaut pour le récepteur. L'interprète est actif, mais ne s'identifie jamais avec les partenaires primaires (1976: 23). Il se trouve hors communication. Il ne doit pas se mêler du contenu du message; son travail consiste à maintenir la communication et à trouver chaque fois une traduction adéquate (1976: 23-24). Kirchoff relève les effets de la proximité ou distance entre les participants. En consécutive l'interprète et les partenaires primaires enregistrent non seulement la dimension verbale, mais aussi visuelle et paraverbale. Il s'agit donc d'une communication plus riche, plus complète. Dans le cas de la simultanée, en revanche, la distance, les cabines et les écouteurs empêchent de percevoir toutes ces dimensions. Il s'agit donc d'une communication plus pauvre. En consécutive il y a une boucle de rétroaction entre l'interprète et ses deux clients; en simultanée, en revanche, il est plus difficile d'avoir cette même boucle, puisque l'interprète est généralement isolé des autres participants (1976: 26-27). Il s'avère donc que certains choix quant au mode d'interpréter et la proximité entre les participants ont des répercussions sur la communication.

Les activités de traduire et d'interpréter sont reprises comme des actes de communication dans les études discursives de Hatim et Mason (1997: 1). Ces auteurs s'intéressent moins à la source et la cible, mais fixent leur attention d'une part sur le traducteur/interprète - qui est vu comme un courtier (1997: 14) - et d'autre part sur le texte, qui est le reflet et le résultat d' un acte de communication. Hatim et Mason distinguent trois domaines textuels, qui peuvent être plus ou moins importants dans les différents modes d'interprétation. Alors que l'interprète consécutif fixe son attention sur la structure, l'interprète simultané la fixe sur la texture, et l'interprète de liaison sur le contexte (1997: 41-42). Ce dernier domaine est plus riche que les domaines de la structure et de la texture, puisqu'il inclut le genre, 
l'intertextualité (1997: 50-51), mais aussi la solidarité, la distance sociale et la proximité physique (1997: 22). Parmi les facteurs contextuels, Hatim et Mason mentionnent également l'intentionnalité, en d'autres mots, la capacité humaine de «faire des choses avec des mots», donc, de produire des actes de langage (1997: 40). On peut en déduire que l'interprète de liaison a le devoir d'empêcher qu'il y ait le moindre doute sur l'intentionnalité des actes de langage qu'il re-produit: l'intentionnalité doit à tout instant être celle du participant dont il traduit le message. Par la voie du contexte, le modèle de la communication s'ouvre donc à la dimension sociologique.

La science de la communication a été lente à s'intéresser à l'interprète interlingual. Parallèlement, les études de la traduction ou de l'interprétation ont été lentes à se rattacher à la science de la communication; ce lien est souvent absent ou implicite. Le modèle de la communication entre trois participants et deux langues accepté généralement est celui de Kirchoff.

Le parcours conceptuel à travers la science de la communication et les études de l'interprétation nous montre que les premiers modèles de la communication par interprète n'ont pas cherché à distinguer immédiatement les modalités simultanée, consécutive et de liaison.

Il convient néanmoins de parler d'un différence considérable sur le plan de la communication. Kirchoff signale que l'interprétation de conférence est plus pauvre sur le plan des langages (paraverbal, gestuel, etc.), puisque l'interprète est isolé dans sa cabine et ne les perçoit pas; l'interprète de liaison, par contre, est soumis à une communication infiniment plus riche en divers langages.

Hatim et Mason, à leur tour, relèvent que l'interprète de liaison fait un travail nettement moins confortable sur le plan de la structure et de la texture, mais que sur le plan du contexte, c'est-à-dire, le plan pragmatique, son travail possède une plus grande richesse.

Finalement, l'interprète en tant que simple médiateur en principe ne s'ingère pas dans le message, ne cherche pas à s'identifier avec ses clients, se tient à distance. Ce principe néanmoins, est plus difficile à respecter pour un interprète de liaison que pour un interprète de conférence. C'est ce qui explique les stratégies de l'interprète social pour maintenir sa neutralité.

\section{Evolution de la linguistique vers la communication et la sociologie: concepts de base}

Après la distinction faite par Saussure entre langue et parole, la linguistique européenne s'est scindée en deux courants. C'est le courant de la parole qui nous intéresse, parce qu'il étudie «l'acte individuel de production (langagière), dans un contexte déterminé, ayant pour résultat un énoncé (...). L'énoncé est le résultat de cet acte, c'est l'acte de création du sujet parlant devenu alors ego ou sujet de l'énonciation» (Dubois et.al. 1994: 180-181).

Cette linguistique tente de dégager les éléments qui, dans les énoncés, constituent des traces du processus d'énonciation qui les a produits. Il s'agit surtout des unités qui ne prennent leur sens qu'à l'occasion d'un acte 
d'énonciation particulier, entre autres les embrayeurs (en anglais 'shifters') ou déictiques. Ces éléments s'articulent autour du lieu, du moment et des participants de l'énonciation. Les embrayeurs de personne peuvent être reliés à l'alignement ('footing') de Goffman, et possèdent donc une dimension sociologique évidente.

Comme pour la sociologie il nous semble intéressant de passer par les réflexions des initiateurs. Tout commence à partir d'un article Jespersen (1947: 23) sur 1' apprentissage des shifters, c'est-à-dire, une catégorie de mots dont la signification dépend de la situation. Apprentissage difficile mais important, puisque les philosophes considèrent l'usage du je comme le premier signe de la conscience de soi. Jakobson approfondit la question des shifters, surtout des déictiques de personne (1971: 132-133).

La problématique est reprise par Benveniste, qui est considéré comme le fondateur de la linguistique de l'énonciation. Son point de départ est justement la problématique de la personne, qui devient rapidement une théorie de l'opposition entre les personnes: je et $t u$. Mais alors que le je et le tu s'impliquent mutuellement dans la corrélation de la personnalité, la troisième personne $i l$ est exclue de leur relation et devient une non-personne (1966: 228). Ce qui différencie le je du $t u$, c'est la subjectivité du je, en d'autres mots, c'est le fait que le je soit toujours inscrit à l'intérieur de l'énoncé, alors que ce n'est pas le cas du tu (1966: 228). Le je et le $t u$ sont définis comme des êtres de discours; le je étant l'individu qui énonce la présente instance de discours contenant l'instance linguistique du je, et le $t u$ étant l'individu allocuté dans la présente instance de discours contentant l'instance linguistique du $t u$ (1966: 253). Benveniste définit la subjectivité comme «la capacité du locuteur à se poser comme sujet», après avoir dit que «c'est dans le langage et par le langage que l'homme se constitue comme sujet» (1966: 259) et «n'est ego qui dit 'ego'» (1966: 60).

L'activité d'interpréter constitue un pierre de touche assez importante pour ces idées. La théorie de la personnalité de Benveniste semble fonctionner dans l'interprétariat (social): il y a en effet plusieurs personnes, notamment le je et le $t u$ qui veulent maintenir un dialogue, mais aussi le $i l$ ou le elle, c'est-à-dire, l'interprète qui les traduit. Selon Benveniste l'interprète serait une non-personne pour ses deux clients.

Quant à la théorie de la subjectivité, le seul sujet possible dans sa théorie semble être le je. En revanche, le 'je' prononcé par l'interprète, qui est une non-personne, toujours il ou elle pour ses clients, malgré le fait d'être un je formel, ne semblerait pas un indice de vraie subjectivité. Le fait de dire 'je' n'est donc pas une condition suffisante pour qu'il y ait un égo, ou du moins, pour qu'on puisse savoir clairement à quelle personne correspond ce 'je'.

En usant les je et les $t u$ qui s'adressent aux interlocuteurs, l'interprète maintient l'interaction originale sans en faire une interaction entre son propre je et les $t u$. Il maintient ainsi toute la personnalité et la subjectivité des interlocuteurs. Quant à lui-même, il évite de devenir une personne ou même une non-personne, en évitant de se transformer en je ou en $t u$, ou même en il ou en elle pendant l'interaction. 
Un développement postérieur de la théorie de l'énonciation, en particulier de la subjectivité, est dû au philosophe et linguiste français Ducrot, qui étudie les convergences de voix dans les conversations ordinaires ou dans des textes littéraires. Ducrot (1984: 171) lance sa recherche dans le but d' attaquer le postulat de l'unicité du sujet parlant. Ses réflexions sur les sujets de l' énonciation sont d'une utilité incontestable pour l'étude de l'énonciation dans une situation d' interprétariat social, où il existe aussi un risque de croire à l'unicité du sujet parlant: en effet, il arrive qu'un client au niveau d'instruction assez bas confonde son interlocuteur et son interprète et s'en prenne à ce dernier, croyant que c'est celui-ci qui a prononcé quelque chose qui lui a déplu.

Ducrot introduit une deuxième distinction (1984: 178-181): il s'agit de la différence entre sens et signification. Alors que le sens est la caractéristique sémantique de l'énoncé, la signification est «l'ensemble des instructions données aux personnes qui ont à interpréter les énoncés de la phrase» (1984: 81). Il est clair que toute opération d'interprétation a comme but non seulement de rendre le sens d'un énoncé, mais aussi de faire très attention à sa signification. Ducrot (1984: 189-190) aborde la question du sujet unique, signalant que les caractéristiques de celui-ci seraient les trois suivantes: être chargé de l'activité psycho-physiologique nécessaire à produire l'énoncé, être l'auteur ou l'origine des actes illocutoires, être désigné à l'intérieur de l'énoncé par la première personne du singulier 'je'.

Ces caractéristiques deviennent problématiques dès qu'il y a la moindre reprise. Pour surmonter ce genre de difficultés, Ducrot a développé sa théorie de la polyphonie, inspirée par la théorie (littéraire) du dialogisme et de la polyphonie de Bakhtine. Le but de Ducrot est d'utiliser les concepts de Bahktine pour distinguer les êtres discursifs qui convergent dans un même énoncé. Sa thèse est qu'il y a au moins deux types de sujets. Le locuteur est l'auteur de l'énoncé, le responsable: le pronom je et d'autres marques de la première personne (1984: 193) se réfèrent à lui. Le sujet parlant est l'auteur empirique, le producteur de l'énoncé (1984: 194). Les deux peuvent coïncider ou être distincts. Le locuteur peut aussi mettre en scène des voix, que Ducrot appelle des énonciateurs (1984: 204-205).

Il aborde également la question de la double énonciation (1984: 197) et du double je qui apparaît par exemple, quand il y a usage du style direct. Ducrot avance qu'il peut y avoir dans un énoncé différents locuteurs, éventuellement subordonnés. Un seul de ces locuteurs peut-être à la fois sujet parlant et locuteur.

Il s'agit chez Ducrot d'une analyse de la subjectivité nettement plus raffinée que chez Benveniste. Ainsi, selon la théorie de Ducrot, l'interprète serait un sujet parlant empirique reprenant dans une autre langue l'énoncé dont un autre (le locuteur) serait l'auteur et le responsable, mais cet interprète n'en serait pas pour autant une non-personne. Pour Ducrot il serait un sujet parmi d'autres sujets, plus ou moins visible selon le cas.

La théorie de Ducrot a connu des développements postérieurs dans quatre ou cinq directions, dont l'une est la théorie Scandinave de la Polyphonie Linguistique (ScaPoLine). Cette théorie, qui est plus abstraite et plus 
stricte que celle de Ducrot, s'intéresse à la relation entre langue et parole sur le plan des traces des locuteurs. Elle s'inspire également de Bahktine. Au sujet des sujets, Nølke dit sans détours que le sujet parlant physique est sans intérêt pour la linguistique, qui ne s'intéresse qu'aux êtres discursifs (2001: 63). Nølke distingue trois types de personnes: le locuteur, l'allocutaire et le tiers (individuel ou collectif); il distingue quatre types de locuteurs, selon les fonctions qu'ils remplissent (auteur de l'événement historique, source, trace d'une activité énonciative antérieure ou ultérieure ou locuteur ayant les aspects d'une personne complète) (2005: 4).

L'analyse polyphonique de Nølke et la ScaPoLine peuvent nous aider à établir une structure polyphonique particulière - par exemple, le fonctionnement des embrayeurs-, ainsi qu'une configuration polyphonique particulière - par exemple, le jeu des embrayeurs dans un texte déterminé(2004: 2). Elles nous offrent une description détaillée du locuteur-en-tantque-constructeur, de l'allocutaire, des points de vue, des êtres discursifs, des tiers, des voix hiérarchiques (ou non), des relations polyphoniques, et de la cohérence polyphonique.

Quant au rôle qui nous intéresse, la piste indiquée par Nølke est la même que celle de Ducrot, notamment le double je du Discours Représenté, phénomène polyphonique par excellence. En effet, Nølke prévoit dans son modèle qu'un locuteur en représente un autre à travers le Discours Direct Rapporté ou le Discours Direct Libre (2004: 61). La différence entre les deux se situe dans la présence ou non d'un inquit, formule par laquelle le locuteur signale préalablement qu'il va rapporter les mots de quelqu'un d'autre (2004: 61). Justement l'interprète social produit d'habitude un double inquit, dirigé à chacun de ses clients, pour expliquer comment fonctionne son travail. Cette formule identifie ce qu'il dira comme un discours rapporté, qui par la suite deviendra un discours rapporté libre. Nølke signale encore qu'en disant qu'il représente quelqu'un d' autre, le locuteur se focalise comme un intermédiaire, qui prend ouvertement la responsabilité de l'analogie entre le discours originel et la représentation (2004: 64). Il s'en suit que l'interprète est bel et bien présent à travers la traduction qu'il produit. Comme il laisse des traces (in)volontaires dans sa traduction, il y a donc lieu de parler de son existence comme personne et comme sujet. Sa voix, bien sûr, ne devient visible que pour ceux qui comparent le texte source et le texte cible.

Nous voyons donc que la linguistique de l'énonciation nous offre des analyses de plus en plus subtiles de la personne et du sujet, dont les études de l'interprétation sociale, par la complexité des situations communicatives et des interactions qu'elles analysent, peuvent tirer un grand profit.

Il est également significatif que Wadensjö se base dans Interpreting as Interaction sur la théorie polyphonique de Bahktine pour suivre l'agencement discursif d' interactions assistées par un interprète social; que Ducrot fasse usage des mêmes concepts pour étudier l'énonciation réalisée par des personnes physiques ou fictives; que Nølke se base sur la même théorie pour étudier les shifters et autres phénomènes discursifs. Il s'agit évidemment de trois usages différents de la même théorie. 
Quant à la subjectivité et la personnalité, Ducrot est intéressé par n'importe quelle énonciation, aussi par les conversations réelles. Les personnes physiques peuvent donc trouver une place dans sa théorie. Les réflexions de Ducrot autour du locuteur portent plutôt sur le(s) sujet(s). Nølke et la ScaPoLine dressent une typologie des personnes linguistiques et des locuteurs (sujets). Ce dernier modèle est plus raffiné ; de plus, il transforme la subjectivité en responsabilité.

Il paraît tout à fait pertinent d'appliquer le modèle de Nølke à l'interprétation (sociale ou autre). C'est sans doute l'analyse polyphonique du Discours Représenté qui offre la voie la plus évidente aux recherches liées à l'interprétation.

\section{Conclusion}

L' interprétation sociale est une interdiscipline naissante. Elle semble surtout inspirée par des concepts sociologiques, alors que la part des concepts issus de la science de la communication et surtout de la linguistique semble moindre. Néanmoins il y a déjà eu des rapprochements importants entre la sociologie, la science de la communication et la linguistique. L'interprétation sociale peut en tirer profit pour développer sa propre théorie.

Notre parcours nous permet de résumer les concepts fondamentaux dans un tableau synoptique. La sociologie en est le point de départ; la science de la communication - sous la forme concrète de l'interprétation sociale- et la linguistique de l'énonciation la complètent. 


\begin{tabular}{|c|c|c|}
\hline $\begin{array}{l}\text { Sociologie: l'action verbale } \\
\text { (termes en français/anglais) }\end{array}$ & $\begin{array}{l}\text { Science de la communication: } \\
\text { la communication verbale }\end{array}$ & $\begin{array}{l}\text { Science du langage: } \\
\text { linguistique } \\
\text { de l'énonciation }\end{array}$ \\
\hline Action, action & $\begin{array}{l}\text { Acte de communication, de } \\
\text { traduire, d'interpréter }\end{array}$ & $\begin{array}{l}\text { Activité d'énonciation (evt. un } \\
\text { discours représenté ou discours } \\
\text { analogue) }\end{array}$ \\
\hline Agent, agent & Participant & $\begin{array}{l}\text { Auteur, responsable de } \\
\text { l'énonciation, } \\
\text { sujet }\end{array}$ \\
\hline Alignement des actants, footing & $\begin{array}{l}\text { Positionnement des participants } \\
\text { primaires et secondaires }\end{array}$ & $\begin{array}{l}\text { Mise en place des (co-) } \\
\text { locuteurs, } \\
\text { interlocuteurs }\end{array}$ \\
\hline $\begin{array}{l}\text { Animator, animator, mediateur } \\
\text { mediator, intermédiaire, go- } \\
\text { between }\end{array}$ & $\begin{array}{l}\text { Pivot, émetteur ou récepteur } \\
\text { secondaire }\end{array}$ & $\begin{array}{l}\text { Co-locuteur, intermédiaire, } \\
\text { locuteur } \\
\text { qui représente un Discours } \\
\text { Représenté }\end{array}$ \\
\hline Audience, audience & $\begin{array}{l}\text { Emetteurs et récepteurs } \\
\text { monolingues }\end{array}$ & Co-locuteur(s) \\
\hline Cadre, framework & Contexte, situation & Discours, texte \\
\hline Face à face, face-to-face & Face à face & $\begin{array}{l}\text { Configuration polyphonique } \\
\text { (le côté physique n'est pas } \\
\text { pertinent) }\end{array}$ \\
\hline $\begin{array}{l}\text { Interaction (dyade, triade), } \\
\text { interaction, performance, } \\
\text { performance }\end{array}$ & $\begin{array}{l}\text { Communication entre deux ou } \\
\text { plus de personnes (dialogue, } \\
\text { trilogue) }\end{array}$ & $\begin{array}{l}\text { Activité co-construite, } \\
\text { (polyphonie) }\end{array}$ \\
\hline $\begin{array}{l}\text { Interaction symbolique, } \\
\text { symbolic interaction }\end{array}$ & $\begin{array}{l}\text { Communication au moyen d'un } \\
\text { code }\end{array}$ & Enonciation \\
\hline Non-personne, non person & $\begin{array}{l}\text { Non-partisan, personne neutre, } \\
\text { hors communication }\end{array}$ & Non-personne, tiers \\
\hline $\begin{array}{l}\text { Rôle, role, p.ex. animateur, } \\
\text { médiateur, animator, mediator }\end{array}$ & $\begin{array}{l}\text { Rôle, p.ex. émetteur primaire } \\
\text { (source), récepteur primaire } \\
\text { (cible), émetteur et récepteur } \\
\text { secondaires (interprète) }\end{array}$ & $\begin{array}{l}\text { Configuration composée par } \\
\text { des } \\
\text { personnes linguistiques: } \\
\text { locuteur, } \\
\text { allocutaire, êtres discursifs }\end{array}$ \\
\hline $\begin{array}{l}\text { Statut dans l'interaction, } \\
\text { position }\end{array}$ & $\begin{array}{l}\text { Place dans le schéma de la } \\
\text { communication }\end{array}$ & $\begin{array}{l}\text { Marque et fonction dans } \\
\text { l' énonciation }\end{array}$ \\
\hline $\begin{array}{l}\text { Soi/Moi, (d'une personne } \\
\text { physique), self }\end{array}$ & $\begin{array}{l}\text { Identité du locuteur (personne } \\
\text { physique) }\end{array}$ & $\begin{array}{l}\text { Sujet, ego, responsable } \\
\text { (personne } \\
\text { physique ou non) }\end{array}$ \\
\hline $\begin{array}{l}\text { Sociation, apprentissage des } \\
\text { formes d'action verbale liées à } \\
\text { la médiation d'un interprète, } \\
\text { sociation }\end{array}$ & $\begin{array}{l}\text { Apprentissage des formes de } \\
\text { communication multilingue au } \\
\text { moyen d'un interprète } \\
\text { Apprentissage de } \\
\text { l'interprétation et du domaine } \\
\text { du contexte }\end{array}$ & $\begin{array}{l}\text { Apprentissage de l'usage des } \\
\text { traces, } \\
\text { p.ex. les déictiques }\end{array}$ \\
\hline
\end{tabular}

\section{Bibliographie}

Anderson, R.B.W. (2002). "Perspectives on the Role of the Interpreter". In Pöchhacker, F.\& M. Schlesinger (2002). 208-217.

Benveniste, E. (1966). Problèmes de linguistique générale I. Paris: Gallimard.

Ducrot, O. (1984). Le dire et le dit. Paris: Editions de Minuit.

Dubois, J., M. Giacomo-Marcellesi \& L. Gespin (1994). Dictionnaire de linguistique et de sciences du langage. Paris: Larousse.

Gentile, A., U. Ozolins \& M. Vasilikakos (1996). Liaison Interpreting. A Handbook. Melbourne: Melbourne University Press. 
Goffman, E. (1959). The Presentation of Self in Everyday Life. Garden City NY: Doubleday Anchor Books.

Goffman, E. (1981). Forms of Talk. Oxford: Blackwell.

Gumperz, J.J. (1982). Language and Social Identity. Cambridge: Cambridge University Press.

Hatim, B. \& I. Mason (1997). The Translator as Communicator. London: Routledge.

Ingram, R. M. (1985). "Simultaneous interpretation of sign languages: semiotic and psycholinguistic perspectives". Multilingua 4(2), 91-102.

Jakobson, R. (1963). Essais de linguistique générale. Paris: Seuil.

Jakobson, R. (1971). Selected Writings II. Word and Language. The Hague/Paris: Mouton.

Jespersen, O. (1947). Language, its Nature, Development and Origin. London: George Allen \& Unwin Ltd.

Kirchoff, H. (1974). Das dreiledige, zweisprachige Kommunikationssystem Dolmetschen. Le Langage et l'Homme 31, 21-27.

Kondo, M. (2000). "3-Party 2-Language Model of Interpreting Research Revisited”. Forum 1 (1), 77-96.

Mead, G.H. (1970). Mind, Self and Society. Chicago/London: the University of Chicago Press.

Nølke, H. (2001). Le regard du locuteur. Paris: Kimé.

Nølke, H., K. Fløttum \& C. Norén (2004). ScaPoLine. La théorie scandinave de la polyphonie linguistique. Paris: Kimé.

Nølke, H. (2005). Dernière évolution de la ScaPoLine. On line sur: www.sens-ettexte.paris4.sorbonne.fr/IMG/pdf/doc-40.pdf.

Pöchhacker, F. \& M. Schlesinger (2002). The Interpreting Studies Reader. London/New York: Routledge.

Pöchhacker, F. (2004). Introducing Interpreting Studies. London/New York: Routledge.

Shannon, C.E. \& W. Weaver (1963). The Mathematical Theory of Communication. Chicago/London: University of Illinois Press.

Wadensjö, C. (1998). Interpreting and Interaction. London/New York: Routledge.

Waters, M. (1994). Modern Sociological Theory. London: Sage. 